\title{
The relationship between anti-vascular endothelial growth factor and fibrosis in proliferative retinopathy: clinical and laboratory evidence
}

\author{
Qi Zhang, ${ }^{1,2}$ Yun Qi, ${ }^{1,2}$ Li Chen, ${ }^{1,2,3}$ Xuan Shi, ${ }^{1,2}$ Yujing Bai, ${ }^{1,2}$ Lvzhen Huang, ${ }^{1,2}$ \\ Wenzhen $\mathrm{Yu}_{\mathrm{u}}{ }^{1,2}$ Yanrong Jiang, ${ }^{1,2}$ Mingwei Zhao, ${ }^{1,2}$ Xiaoxin $\mathrm{Li}^{1,2}$
}

- Additional material is published online only. To view please visit the journal online (http://dx.doi.org/10.1136/ bjophthalmol-2015-308199).

\section{${ }^{1}$ Department of Ophthalmology, Peking University People's Hospital, Key Laboratory of Vision Loss and Restoration, Ministry of Education, Beijing, China ${ }^{2}$ Beijing Key Laboratory for the Diagnosis and Treatment of Retinal and Choroid Diseases, Beijing, China \\ ${ }^{3}$ Ophthalmology Department, Xian No. 4 Hospital, Xincheng District, Xi'an, China}

\section{Correspondence to}

Dr Xiaoxin Li, Key Laboratory of Vision Loss and Restoration, Ministry of Education, Department of Ophthalmology, Peking University People's Hospital, Xizhimen South Street 11, Xi Cheng District, Beijing 100044, China; drlixiaoxin@163.com

QZ, YQ, LC and XS contributed equally and are cofirst authors.

Received 3 December 2015 Revised 2 June 2016 Accepted 3 June 2016 Published Online First 16 August 2016

CrossMark

To cite: Zhang Q, Qi Y,

Chen $\mathrm{L}$, et al. Br J

Ophthalmol

2016:100:1443-1450.

\section{ABSTRACT}

Purpose To investigate the progression of epiretinal membranes after intravitreal bevacizumab (IVB) injection therapy in patients with proliferative membranes and evaluate the changes in fibrosis-related cytokines in retinal pigment epithelial cells and glial cells after treatment with bevacizumab.

Methods Retrospective study of the proliferative membranes in patients with and without IVB therapy. In vitro, the human adult retinal pigment epithelial (ARPE-19) cells and BV2 microglial cell lines were incubated in different bevacizumab concentrations under hypoxic conditions. Cell culture supernatants and cell lysates were harvested after incubation for 24,48 or 72 hours for ELISA and western blot.

Results Bevacizumab accelerated fibrosis in patients with proliferative membranes. Immunofluorescence analysis revealed more intense transforming growth factor $\beta 2$ (TGF $\beta 2$ ) and connective tissue growth factor (CTGF) staining in IVB-treated proliferative diabetic retinopathy (PDR) membranes compared with membranes of patients not receiving IVB therapy. This result was consistent with real-time PCR results. Bevacizumab incubation significantly upregulated TGF $\beta 2$ and CTGF in ARPE-19 cells and BV2 microglial cells, but ciliary neurotrophic factor (CNTF) expression was upregulated only in BV2 microglial cells.

Conclusions Anti-vascular endothelial growth factor treatment likely accelerates fibrosis in PDR patients via upregulation of TGF $\beta 2$, CTGF and CNTF, suggesting the importance of adjunctive therapy for retinal fibrosis.

\section{INTRODUCTION}

Retinal neovascularisation occurs in common diseases, such as diabetic retinopathy (DR), retinopathy of prematurity (ROP) and central retinal vein occlusion, which are responsible for vision loss worldwide. ${ }^{1}$ Vascular endothelial growth factor (VEGF) is a key factor in the pathogenesis of intraocular vascular diseases. VEGF is released in response to hypoxia and elicits neovascularisation and vascular hyperpermeability. ${ }^{2}$ Therapeutic interventions that affect VEGF include intraocular neutralisation and capture by chimeric receptors or antibodies. Recent findings from laboratories and clinical trials demonstrate that VEGF inhibition is a potent therapeutic strategy for retinal neovascular diseases. $^{3-6}$

Bevacizumab, a pan anti-VEGF antibody, has widely been used as an off-label intraocular injection drug in the management of retinal vascular diseases, especially neovascular age-related macular degeneration (AMD), ${ }^{4} \mathrm{ROP}^{3}$ and diabetic macular oedema. ${ }^{5}$ Recently, intravitreal bevacizumab (IVB) injection has been administered to patients with proliferative DR (PDR) before vitrectomy to reduce the risk of intraoperative bleeding. ${ }^{7}$ Although IVB therapy provides significant treatment benefits in multiple indications, not all patients achieve an optimal response, as some patients experience the development and progression of tractional retinal detachment, retinal pigment epithelium tearing and accelerate subretinal fibrosis. ${ }^{8} 9$

Retinal pigment epithelial cells and retinal glial cells play key roles in neovascular retinopathy via the production of angiogenic, fibrotic and inflammatory factors. ${ }^{10}{ }^{11}$ Both of these cell types have been reported to participate in the process of fibrosis in PDR or during subretinal fibrosis formation. Several recent studies reported that IVB therapy contributes to an angiofibrotic switch in neovascularisation retinopathy by upregulating transforming growth factor- $\beta 2$ (TGF $\beta 2$ ), connective tissue growth factor (CTGF) and other effectors. ${ }^{12} 13$ However, these studies primarily focused on clinical patient samples, such as vitreous humour. The potential effect of IVB therapy on fibrosis progression has not been fully explored.

In the present study, we focused on the clinical characteristics of patients with PDR after IVB treatment and detected the expression of TGF $\beta 2$, CTGF and the growth factor ciliary neurotrophic factor (CNTF) in patient membranes. For in vitro analyses, we used the human adult retinal pigment epithelial (ARPE-19) cells and microglia to investigate the changes in fibrosis-associated cytokines, including TGF 32 , CTGF and CNTF, after bevacizumab treatment under hypoxic conditions. The findings of the present study enriched our understanding of the relationship between the development and progression of retinal fibrosis and anti-VEGF therapy.

\section{METHODS \\ Human material}

The Ethical Committee and Institutional Review Board of Peking University People's Hospital (Beijing, China) approved the human patient study protocol, which was conducted in accordance with the Declaration of Helsinki. Written informed consent was obtained from each study subject.

The medical records of patients who were diagnosed with PDR (stage 5) and received IVB injections (Avastin; Genentech; off-label usage, $1.25 \mathrm{mg}$ for patients with PDR) at the Department of 
Table 1 Demographics of the patients with proliferative diabetic retinopathy

\begin{tabular}{|c|c|c|c|c|c|c|c|}
\hline Patient & Gender & $\begin{array}{l}\text { Age } \\
\text { (years) }\end{array}$ & $\begin{array}{l}\text { Mean duration } \\
\text { of diabetes } \\
\text { (years) }\end{array}$ & $\begin{array}{l}\text { Mean fasting } \\
\text { blood glucose }\end{array}$ & $\begin{array}{l}\text { Vitreous } \\
\text { haemorrhage }\end{array}$ & $\begin{array}{l}\text { Fibroneovascular } \\
\text { membranes }\end{array}$ & $\begin{array}{l}\text { Tractional } \\
\text { retinal } \\
\text { detachment }\end{array}$ \\
\hline 1 & $M$ & 55 & 14 & 8.5 & + & + & - \\
\hline 2 & $M$ & 51 & 9 & 9.2 & + & + & - \\
\hline 3 & $\mathrm{~F}$ & 53 & 11 & 7.8 & + & + & - \\
\hline 4 & $\mathrm{~F}$ & 62 & 16 & 7.0 & + & + & - \\
\hline
\end{tabular}

Ophthalmology, Peking University People's Hospital (Beijing, China) were retrospectively reviewed from August 2012 to September 2014. Patients with subsequent progression of membrane fibrosis based on examinations after IVB treatment were selected. A total of four eyes of four patients with PDR (table 1) were identified for image acquisition, and their medical records were reviewed.

Immunofluorescence (IF) staining and real-time PCR assay were used to evaluate the expression and concentration of different cytokines. A total of 11 samples were collected from patients with PDR after 3 weeks of IVB treatment, and 11 membranes from patients with PDR not receiving IVB treatment were collected as controls. All of the patients with PDR are type 2 diabetes mellitus, and the general physical information, including age, gender, body mass index and blood glucose level did not vary significantly between the two groups. Dr Xiaoxin $\mathrm{Li}$ and Dr Yanrong Jiang performed IVB therapy and the vitrectomy surgeries in these patients. All membranes from patients with PDR were immediately stored at $-80^{\circ} \mathrm{C}$ until analysis.

\section{IF staining}

IF staining was performed on 14 PDR membranes, which are seven samples from IVB treatment group, and seven samples from non-treatment group in $10 \mu \mathrm{m}$ thick frozen sections. We used antibodies against the following antigens: TGF $\beta 2$ (1:200 dilution, ab113670, Abcam, Cambridge, Massachusetts, USA), CTGF (1:200 dilution, ab66218, Abcam), CNTF (1:200 dilution, ab66218, Abcam), VEGF (1:200 dilution, ab1316, Abcam) and vimentin (1:150 dilution, Zhongshan Golden Bridge Biotechnology, Beijing, China). Samples were counterstained with DAPI (1:1000 dilution, D9542, Sigma-Aldrich, USA). Sections were examined and photographed using a fluorescence microscope (DS-Ril-U2, Nikon, Tokyo, Japan).

\section{Membrane RNA extraction and real-time PCR}

Four membranes of four patients with PDR not receiving IVB therapy (control group) and four membranes of four patients who underwent previous IVB treatment (IVB treatment group) were used for real-time PCR assays. The membranes were lysed in TRIzol reagent (Invitrogen, Carlsbad, California, USA). Reverse transcription reactions were performed using a RevertAid First Strand cDNA Synthesis kit with oligo-dT primers (Fermentas, Pittsburgh, Pennsylvania, USA). Real-time PCR was performed using the SYBR Green PCR mix (Thermo, Pittsburgh, Pennsylvania, USA) and an ABI7300 real-time PCR system (Applied Biosystems, Life Technologies, Foster City, California, USA). The following PCR conditions were used: 30 cycles of denaturation at $94^{\circ} \mathrm{C}$ for $15 \mathrm{~s}$, annealing for $30 \mathrm{~s}$ at $60^{\circ}$ $\mathrm{C}$ for human TGF 32 and human glyceraldehyde 3-phosphate dehydrogenase (GAPDH) or at $63^{\circ} \mathrm{C}$ for human CTGF and extension at $72^{\circ} \mathrm{C}$ for $2 \mathrm{~min}$, followed by an extension step at $70^{\circ} \mathrm{C}$ for $7 \mathrm{~min}$. Table 2 shows the primers used for real-time
Table 2 Human primers for real-time PCR

\begin{tabular}{|c|c|c|}
\hline Primer & Sequence $\left(5^{\prime}\right.$ to $\left.3^{\prime}\right)$ & Product size \\
\hline TGF $\beta 2$ & $\begin{array}{l}\text { Forward GTTCGATTTGACGTCTCAGCAAT } \\
\text { Reverse CAATCCGTTGTTCAGGCACTCT }\end{array}$ & 107 \\
\hline CTGF & $\begin{array}{l}\text { Forward CTCGCGGCTTACCGACTG } \\
\text { Reverse GGCTCTGCTTCTCTAGCCTG }\end{array}$ & 169 \\
\hline CNTF & $\begin{array}{l}\text { Forward CGTTGGAGACATACACAAATGG } \\
\text { Reverse AGAACCCTGAAGTGGAAGGAC }\end{array}$ & 126 \\
\hline VEGF & $\begin{array}{l}\text { Forward ACTGAGGAGTCCAACATCAC } \\
\text { Reverse CTTGTCTTGCTCTATCTTTC }\end{array}$ & 130 \\
\hline GAPDH & $\begin{array}{l}\text { Forward GAGTCCACTGGCGTCTTCAC } \\
\text { Reverse GITCACACCCATGACGAACA }\end{array}$ & 120 \\
\hline
\end{tabular}

CNTF, ciliary neurotrophic factor; CTGF, connective tissue growth factor; GAPDH, glyceraldehyde 3-phosphate dehydrogenase; TGF, transforming growth factor; VEGF, vascular endothelial growth factor.

PCR. The data were normalised to the level of the housekeeping gene human GAPDH, and the relative fold changes in mRNA expression were determined by calculating the $2^{-\Delta \Delta \mathrm{ct}}$. Each experiment was repeated five times.

\section{Cell culture and drug treatment}

ARPE-19 cells and BV2 microglial cells were obtained from the American Type Culture Collection (ATCC, Manassas, Virginia, USA). The ARPE-19 cells were cultured in Dulbecco's modified Eagle's medium/F12 medium (HyClone; Hyclone, Grand Island, New York, USA) supplemented with 10\% fetal bovine serum (FBS; Invitrogen-Gibco, Grand Island, New York, USA). The BV2 microglial cells were cultured in RPMI 1640 medium supplemented with $10 \%$ FBS and $5 \mathrm{mM}$ glucose. Both sera contained $1 \%$ penicillin (100 units $/ \mathrm{mL})$ and streptomycin $(100 \mu \mathrm{g} / \mathrm{mL})$. Cells were cultured at $37^{\circ} \mathrm{C}$ under hypoxic conditions $\left(1 \% \mathrm{O}_{2}, 5 \% \mathrm{CO}_{2}\right)$. The cells were seeded in six-well plates at a density of $5 \times 10^{5}$ cells per well in $2000 \mu \mathrm{L}$ of complete culture medium. After proliferation for 24 hours, the cells were independently treated with different concentrations $(0.125$, $0.25,0.5,1$ or $2 \mathrm{mg} / \mathrm{mL}$ ) of bevacizumab (Roche, China). Bevacizumab is the first commercially available angiogenesis inhibitor that blocks angiogenesis by inhibiting all forms of VEGF-A, and it is humanised from the murine-neutralising antibody. ${ }^{14}$ The cells were cultured for an additional 24,48 or 72 hours under hypoxic conditions.

\section{Cell proliferation assay}

Cell proliferation assays were performed as described previously. ARPE-19 cells and BV2 microglial cells were incubated in 96-well plates. Cell Counting Kit-8 (CCK-8; Dojindo, Shanghai, China) assays were performed according to the manufacturer's instructions, and the absorbance was read using an ELISA microplate reader (Finstruments Multiskan Model 347; MTX 
Lab Systems, Vienna, Virginia, USA). Each experiment was performed in five wells and repeated at least three times.

\section{TGFB2, CTGF and CNTF measurement using ELISA}

The same treatment process as that for the proliferation assay was used for ELISA. Cell culture supernatants were harvested after incubation for 24, 48 or 72 hours and were centrifuged for $15 \mathrm{~min}$ at $1000 \mathrm{~g}$. Free TGF 32 , CTGF and CNTF in the culture media were measured using human TGFß2 (DB250, Quantikine HS, R\&D Systems, Minneapolis, Minnesota, USA), human CTGF (SEA010Hu, USCN, Life Science, Wuhan, China) and human CNTF ELISA kits (DNT00, Quantikine HS, R\&D Systems, Minneapolis, Minnesota, USA), respectively, as instructed by the manufacturer. A standard curve was generated using positive protein standards for each assay. Each experiment was performed in five wells and repeated at least three times.

\section{Protein extraction and western blot analysis of ARPE-19 cells and BV-2 cells}

ARPE-19 cells and BV-2 cells were treated with different bevacizumab concentrations $(0.125,0.25,0.5,1.0$ or $2.0 \mathrm{mg} / \mathrm{mL})$, cultured for 24, 48 or 72 hours under hypoxic conditions, and washed with ice-cold PBS. Then, lysates were prepared using RIPA protein extraction buffer (1\% Nonidet P-40, 0.5\% sodium deoxycholate and $0.1 \%$ SDS in PBS) supplemented with a protease inhibitor cocktail (Pierce, Rockford, Illinois, USA). The lysates were cleared via centrifugation at $15000 \mathrm{~g}$ for $15 \mathrm{~min}$ at $4^{\circ} \mathrm{C}$. The supernatant was collected, and the protein content of each lysate was measured using a BCA protein assay kit (Pierce, Rockford, Illinois, USA) according to the manufacturer's instructions.

Equal amounts of protein were separated via 10\% SDS-PAGE and electrophoretically transferred to polyvinylidene difluoride membranes (Amersham, Little Chalfont, UK). The membranes were blocked with $5 \%$ non-fat milk for 1 hour and then incubated overnight at $4^{\circ} \mathrm{C}$ in the following primary antibodies: rabbit anti-CNTF (1:1000, ab110015, Abcam), rabbit anti-CTGF (1:1000, ab6992, Abcam), rabbit anti-TGF $\beta 2$ $(1: 1000$, ab113670, Abcam) and rabbit anti- $\beta$-actin $(1: 1000$, \#4970, Cell Signaling Technology, USA). Next, the membranes were incubated in a goat anti-rabbit horseradish peroxidaseconjugated secondary antibody $(1: 3000, \# 7074$, Cell Signaling Technology) for 1 hour at room temperature. Proteins were visualised using enhanced chemiluminescence western blot detection reagents (Pierce, Rockford, Illinois, USA). The density of each band was analysed using ImageJ software. Western blot analyses were repeated three times, and qualitatively similar results were obtained from each replicate.

\section{Statistical analysis}

Data analysis was performed using the following statistical software programs: Prism V.5 (GraphPad Software, San Diego, California, USA) and SPSS (V.17.0; SPSS, Chicago, Illinois, USA). All data are presented as the means \pm SEM, and the normality of distributions was assessed. Individual group means were compared using Student's unpaired t-test, and data sets were examined via one-way analysis of variance followed by post hoc analysis using Dunnett's test. A two-tailed $\mathrm{p}$ value $<0.05$ was considered to indicate a statistically significant difference.

\section{RESULTS}

IVB injections accelerate fibrosis in patients with proliferative retinopathy: representative case reports

A 55-year-old man reported vision loss in both eyes for 1 year, and he was diagnosed with PDR. IVB therapy was performed to diminish the neovascularisation. After 3 weeks of therapy, membrane proliferation had progressed, and the patient underwent pars plana vitrectomy. B scan images (figure 1) captured before IVB treatment revealed proliferative epiretinal membranes in the superior portion of the retina and in the macular and optic disc section of the left eye. After the initial 3 weeks of IVB
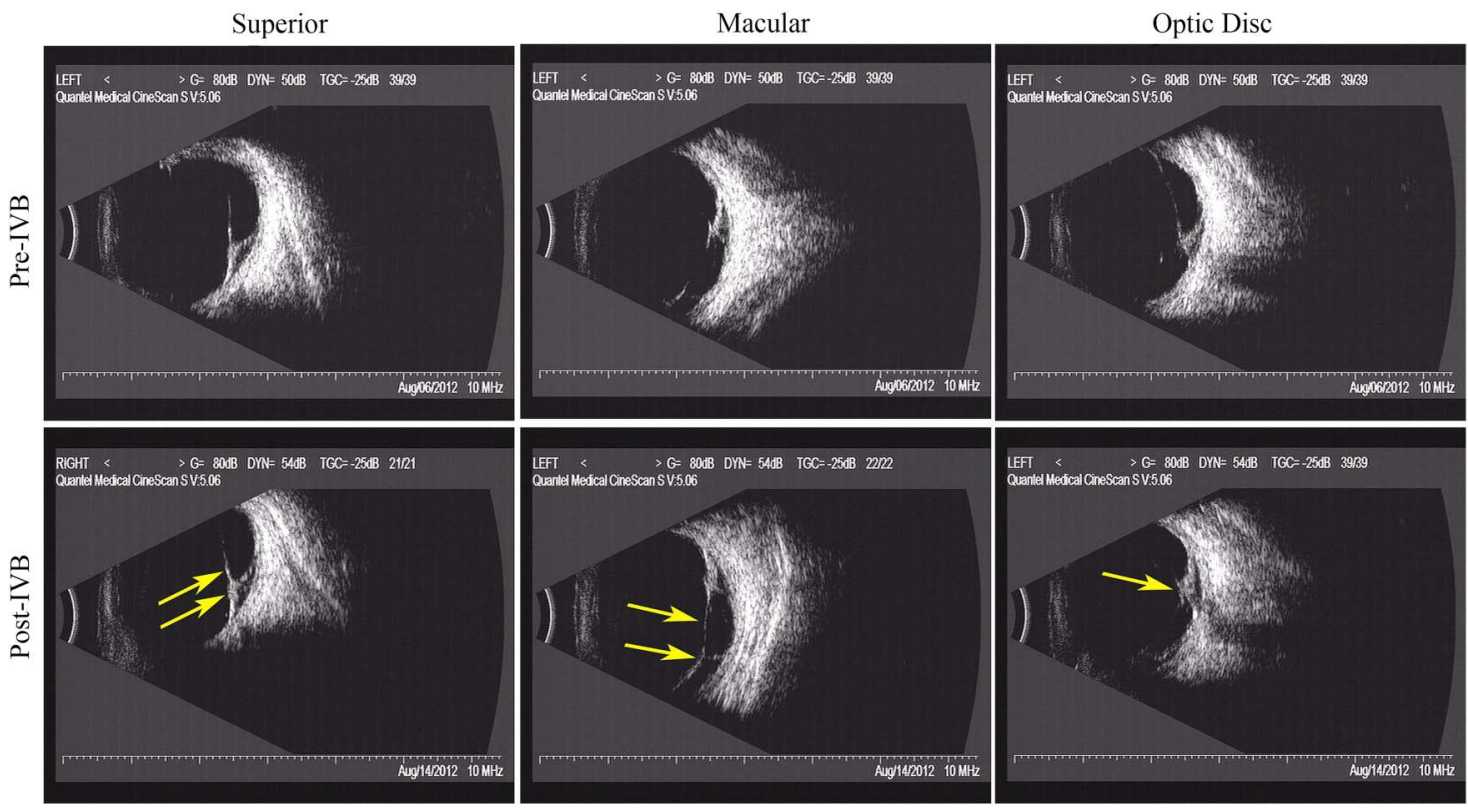

Figure 1 B scan images of a representative patient with proliferative diabetic retinopathy (PDR). B scanning was performed before and after intravitreal bevacizumab (IVB) injection therapy in a patient with PDR. The images show the presence of retinal fibrosis in the superior, macular and optic disc regions of the retina before treatment. However, retinal fibrosis progressed after the initial 3 weeks of IVB treatment. The arrows show the progression of epiretinal membranes compared with the pretreatment conditions. 
treatment, the membranes had progressed significantly compared with the pretreatment conditions in each region of the retina (figure 1, arrows indicate the proliferation part of each region).

TGF $\beta 2$ and CTGF were strongly expressed in the membranes of IVB-treated patients with PDR

Vimentin, TGF 32 , CTGF, CNTF and VEGF were positively stained in all fibrotic membrane specimens (figure 2A-D). However, more intense TGF 32 (figure $2 \mathrm{~A}$, IVB treatment group) and CTGF staining (figure $2 \mathrm{~B}$, IVB treatment group) was detected in the IVB-treated membranes than that in the untreated membranes (figure 2A, B, no treatment group). Alternatively, VEGF staining was weaker in the IVB-treated group than that in the untreated group (figure $2 \mathrm{C}$ ). No apparent changes in CNTF expression were observed between the two groups.

TGF 32 and CTGF mRNA expression was significantly higher in the membranes of the patients in the IVB treatment group than in those of the patients in the untreated group (figure $2 \mathrm{E}$ ). As expected, VEGF mRNA expression was downregulated in the IVB treatment group, but no change in CNTF mRNA expression was observed.

TGF $\beta 2$ and CTGF were upregulated in the supernatants of ARPE-19 cells and BV2 microglial cells

Secreted TGF $\beta 2$ and CTGF were detected in the ARPE-19 cells and BV2 microglial cell supernatants using ELISA kits after incubation in bevacizumab under hypoxic culture conditions. No significant change in the expression of TGF $\beta 2$, CTGF or CNTF (data not shown) was observed in these supernatants after incubation in bevacizumab for 24 or 48 hours. After treatment for 72 hours, both TGF $\beta 2$ and CTGF were upregulated in the cells treated with $0.125,0.25$ or $0.5 \mathrm{mg} / \mathrm{mL}$ bevacizumab (figure 3). However, at higher bevacizumab concentrations $(1.0$ or $2.0 \mathrm{mg} / \mathrm{mL})$, the secreted TGF $\beta 2$ and CTGF levels were reduced (figure $3 \mathrm{~A}, \mathrm{~B}, \mathrm{D}, \mathrm{E}$ ).

TGFB2 and CTGF were upregulated in ARPE-19 cells and BV2 microglial cell lysates

As shown in figure 4, TGF $\beta 2$ was upregulated in ARPE-19 cells after incubation in different bevacizumab concentrations. TGF $\beta 2$ expression was most significantly altered within the first 24 hours of incubation in bevacizumab, was slightly reduced during the following 48 hours and was diminished after 72 hours of treatment with bevacizumab (figure 4A). CTGF expression was significantly upregulated at 48 hours of bevacizumab treatment but was reduced at 72 hours of bevacizumab treatment (figure 4B).

In BV2 microglial cells, treatment with $0.5 \mathrm{mg} / \mathrm{mL}$ bevacizumab for 24 hours significantly induced TGF $\beta 2$ upregulation, but this effect was reduced following 48 and 72 hours of bevacizumab treatment. Alternatively, incubation in 0.125 or $0.25 \mathrm{mg} / \mathrm{mL}$ bevacizumab induced high TGF 32 expression at 48 and 72 hours (figure 5A). CTGF expression peaked after 24 hours of
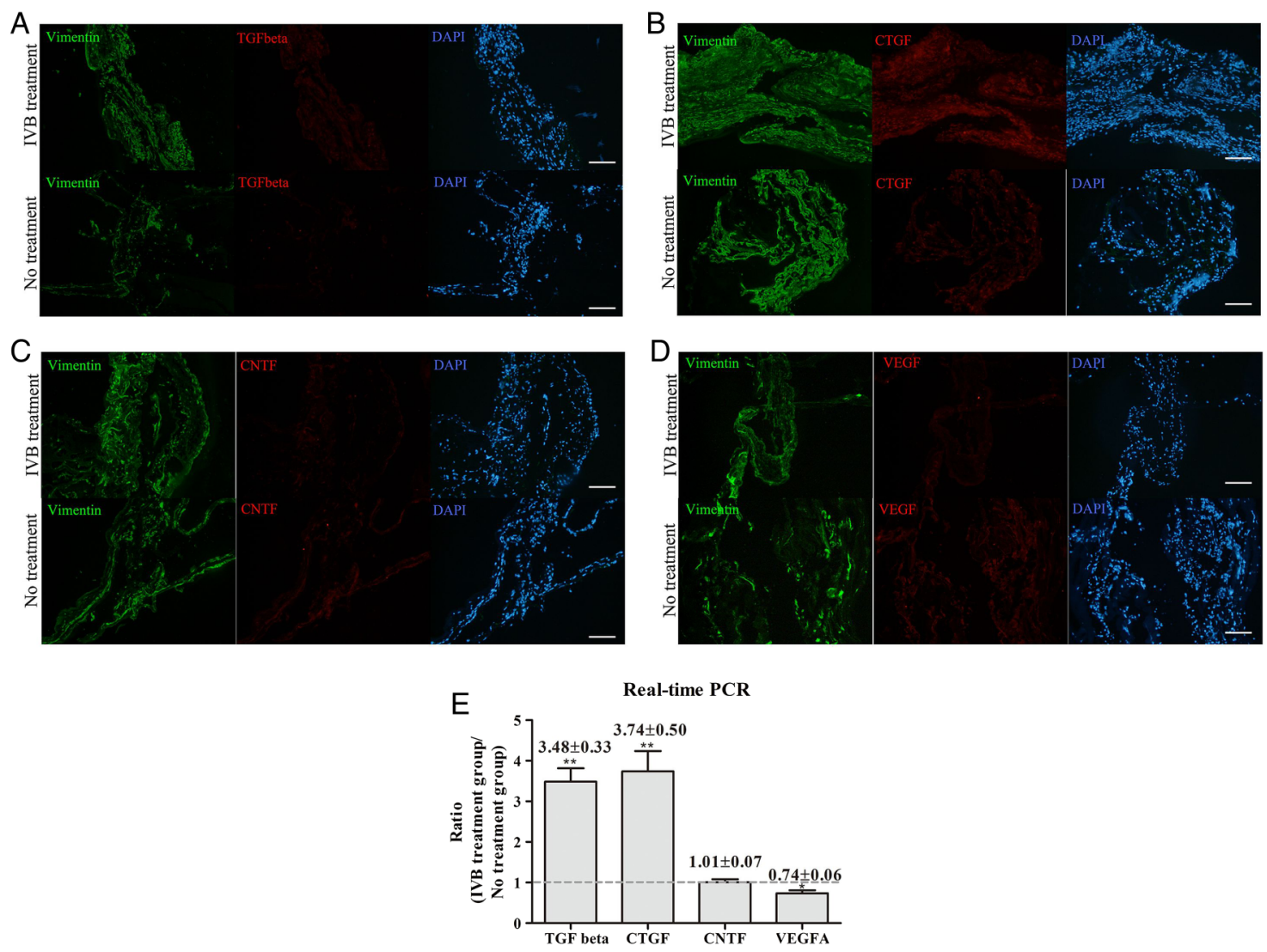

Figure 2 Immunofluorescence staining of epiretinal membranes. Besides vimentin and DAPI staining, panels (A-D) show staining for transforming growth factor $\beta 2$ (TGF 32 ), connective tissue growth factor (CTGF), ciliary neurotrophic factor (CNTF) and vascular endothelial growth factor (VEGF), respectively, in membranes of untreated patients or patients treated with post-intravitreal bevacizumab (IVB) injection. (E) shows the real-time PCR results of TGF $\beta 2$, CTGF, CNTF and VEGF expression in the post-IVB-treated membranes compared with the untreated membranes. The data are presented as the means \pm SEM. ${ }^{*} p<0.05,{ }^{* *} p<0.01$. 

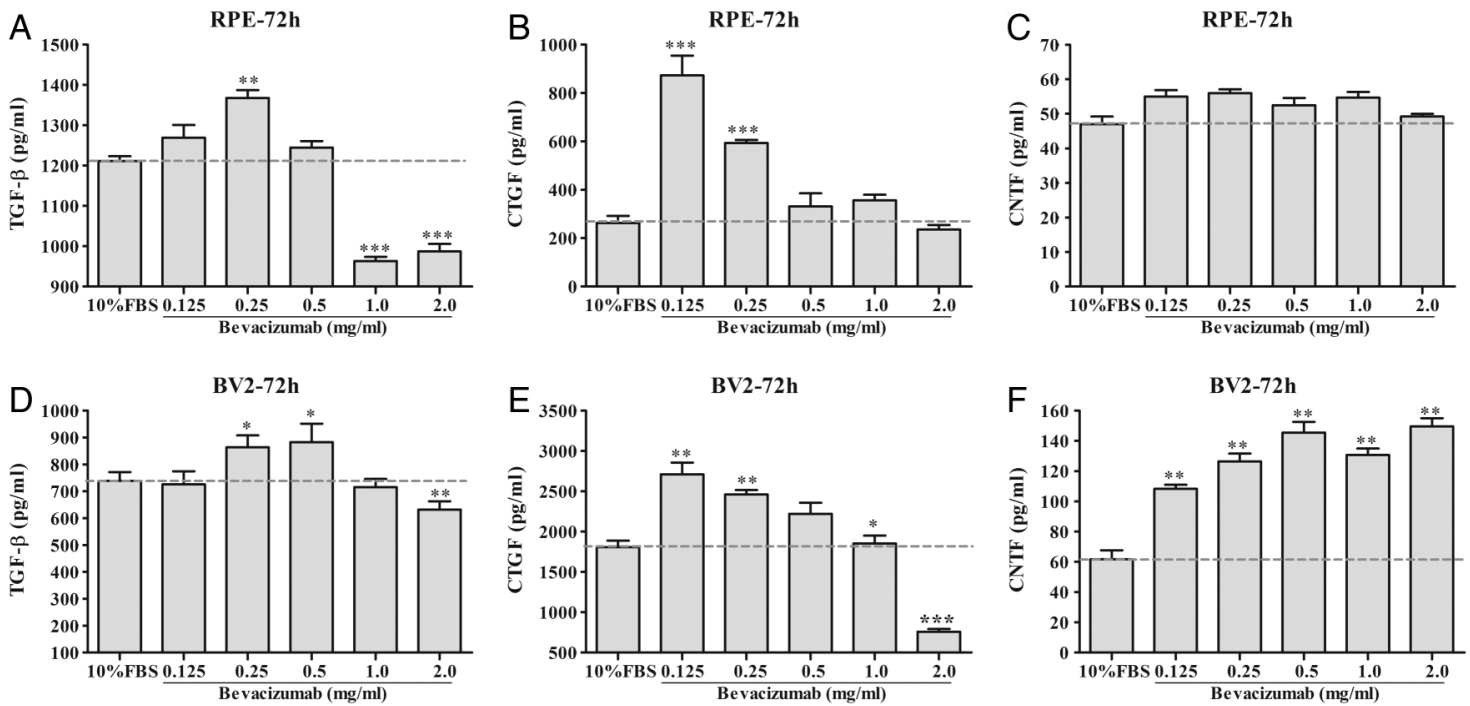

Figure 3 ELISA results of the transforming growth factor $\beta 2$ (TGF $\beta 2$ ), connective tissue growth factor (CTGF) and ciliary neurotrophic factor (CNTF) levels in ARPE-19 and BV2 microglial cell supernatants. The concentrations of free TGF $\beta 2$, CTGF and CNTF in the supernatants of ARPE-19 cells and BV2 microglial cells were measured using ELISA kits. Statistical analyses of the free cytokines measured using ELISA at 72 hours are presented.

Panels (A-C) show the results of TGF $\beta 2$, CTGF and CNTF, respectively, in ARPE-19 cell supernatants. Panels (D-F) show the results of TGF $\beta 2$, CTGF and CNTF, respectively, in BV2 microglial cell supernatants. Each experiment was performed in six wells and was repeated at least three times. The data are presented as the means \pm SEM. ${ }^{*} p<0.05,{ }^{* *} p<0.01,{ }^{* * *} p<0.0001$.

incubation in different bevacizumab concentrations and decreased thereafter (figure 5B).

\section{CNTF was upregulated in BV2 microglial cell supernatants and cell lysates}

As shown in figures 4 and 5, CNTF expression was not upregulated in response to bevacizumab in ARPE-19 cell supernatants or cell lysates (figures 3C and 4C). However, after incubation in bevacizumab for 48 hours, the BV2 microglial cells exhibited significant CNTF expression (figure 5C). The expression level of CNTF in BV2 microglial cell supernatants was upregulated after 72 hours of bevacizumab treatment compared with nontreatment (figure $3 \mathrm{~F}$ ).

\section{DISCUSSION}

There are three main findings in the present study. First, we reported that IVB treatment induced progression in representative patients with PDR (stage 5). Second, we detected more intense TGF 22 and CTGF staining in epiretinal fibrosis PDR membranes treated with IVB than in untreated PDR membranes. Finally, we demonstrated that bevacizumab incubation induced the upregulation of TGF 32 and CTGF in both supernatants and cell lysates of ARPE-19 cells and BV2 microglial cells and that the upregulation of CNTF was induced only in BV2 microglial cells.

Post-bevacizumab fibrotic phenomena were observed previously in patients with $\mathrm{PDR}^{15}$ and $\mathrm{AMD}^{9}{ }^{16}$. In the present study, we also demonstrated that in representative clinical PDR (stage 5), IVB treatment accelerated the progression of epiretinal membranes (figure 1). PDR is a complication of DR that may cause blindness. Although panretinal photocoagulation is one treatment for PDR, this treatment may affect vision as a secondary effect. ${ }^{6}$ Martinez-Zapata $e t a l^{7}$ recently assessed the effectiveness and safety of anti-VEGF drugs for PDR in randomised controlled trials. The results suggested that anti-VEGF drugs reduce the risk of intraocular bleeding in people with PDR. However, based on our findings and other reports, ${ }^{13} \quad 17 \quad 18$ bevacizumab treatment increased the degree of fibrosis, resulting in the need for vitreoretinal surgery. Furthermore, Arevalo et $a l^{17}$ noted that tractional retinal detachment occurred after a mean of 13 days after bevacizumab treatment, and their observation is consistent with our findings that the progression of epiretinal membranes occurred after approximately 3 weeks of bevacizumab treatment.

The elevation of TGF $\beta 2^{12}$ and CTGF ${ }^{13} 19$ has been reported to positively correlate with the formation and progression of epiretinal fibrotic membranes. However, most of these studies only examined the expression of the above factors in vitreous body fluid. Our study revealed that TGF 32 and CTGF exhibited strong staining in IVB-treated membranes after 3 weeks of bevacizumab treatment (figure 2A, B). As demonstrated previously, TGF $\beta$ is a multifunctional cytokine that regulates critical cell biological responses, such as differentiation, apoptosis and migration. TGF $\beta$ is believed to be the most important ligand in the pathogenesis of fibrotic diseases in the retina, including proliferative vitreoretinopathy (PVR), PDR and ROP. ${ }^{20} 21$ In addition, TGF $\beta$ contributes to the contraction of epiretinal and subretinal membranes in patients with PDR and PVR. ${ }^{21}$ Specifically, the concentration of TGF $\beta 2$, which is the predominant TGF $\beta$ isoform, significantly correlates with the severity of PVR. ${ }^{22} 23$ Previous reports studied the expression of CTGF in epiretinal fibrovascular membranes from patients with PDR. CTGF is a secreted protein that modulates the activity of many growth factors and extracellular matrix (ECM) proteins, leading to tissue reorganisation such as ECM formation and remodelling, wound healing and fibrosis. ${ }^{24}$ Immunohistochemical study demonstrated that CTGF was expressed by endothelial cells and myofibroblasts in PDR epiretinal membranes. ${ }^{25}$ CTGF is also induced by growth factors such as VEGF and TGF $\beta .{ }^{24}$ In PDR, the switch from neovascularisation to a fibrotic phase, termed the angiofibrotic switch, is mediated by CTGF, which maintains a critical balance with VEGF. ${ }^{19} \mathrm{Abu}$ El-Asrar $e a^{26}$ also demonstrated significantly higher levels of CTGF in vitreous samples from patients with active PDR angiogenesis compared with 
A
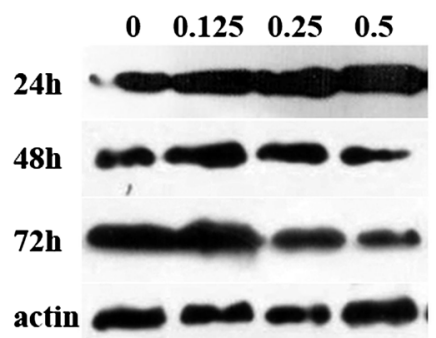

B

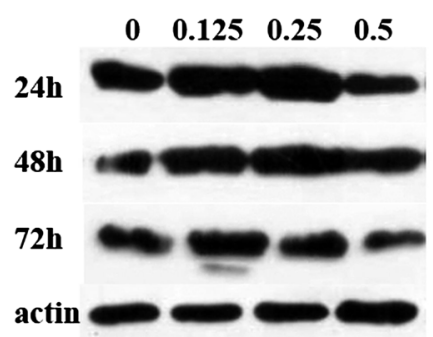

C

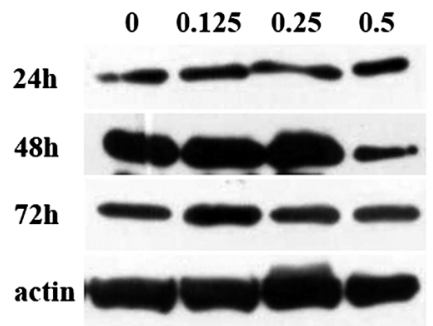

RPE TGF beta

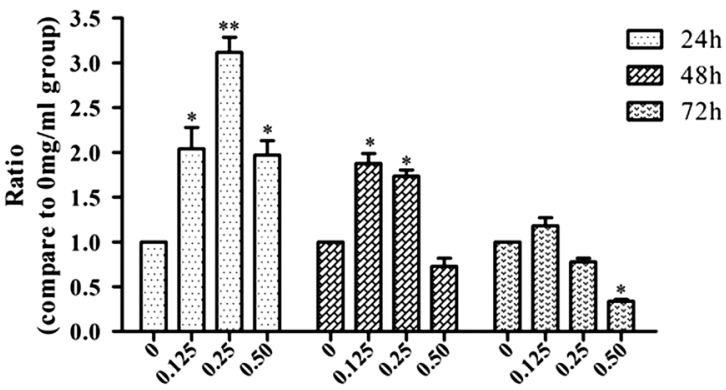

RPE CTGF

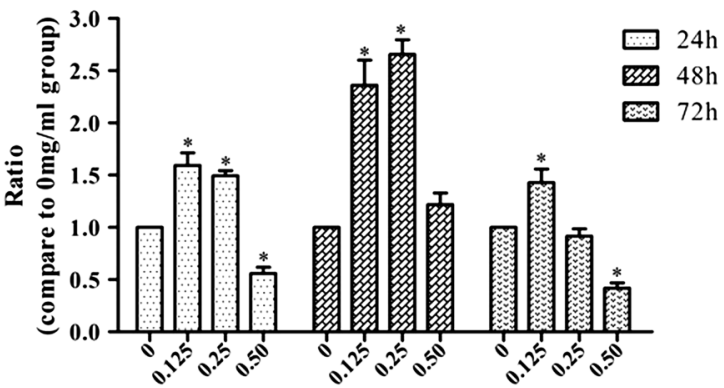

RPE CNTF

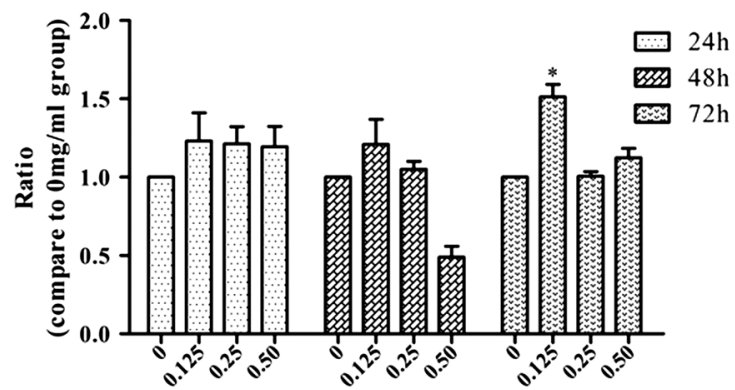

Figure 4 Western blot results of (A) transforming growth factor $\beta 2$ (TGFB2), (B) connective tissue growth factor (CTGF) and (C) ciliary neurotrophic factor (CNTF) expression in ARPE-19 cell lysates. Immunoblot images and statistical analyses for TGF 32 , CTGF and CNTF are presented. The results show that after intravitreal bevacizumab (IVB) injection, TGF $\beta 2$ and CTGF were upregulated after 24 and 48 hours but were reduced at 72 hours. However, no apparent change in CNTF expression was observed at any time point. Western blot analyses were repeated three times, and qualitatively similar results were obtained in each replicate. The data are presented as the means \pm SEM. ${ }^{*} p<0.05,{ }^{* *} p<0.01$.

inactive PDR. One recent study suggested that the continued production of excess CTGF activates the angiofibrotic switch, which triggers fibrosis, consequent scar formation and vision loss. ${ }^{27}$ Previously published data and our findings suggest that TGF $\beta 2$ and CTGF participate in the process of retinal fibrosis after IVB therapy.

However, the source of TGF $\beta 2$ and CTGF secretion after IVB treatment remains uncertain. Previously, Min Zhang et al ${ }^{12}$ demonstrated that HUVECs upregulate the expression of CTGF, bFGF, TGF $\beta 2$ and MMP-2, which are involved in fibrosis after anti-VEGF therapy. In the present study, we demonstrated that anti-VEGF induces the upregulation of TGF 32 and CTGF in ARPE-19 cells and microglial supernatants and cell lysates. High concentrations of bevacizumab $(1.0$ or $2.0 \mathrm{mg} / \mathrm{mL})$ did not induce TGF $\beta 2$ or CTGF secretion, because of the inhibition of the proliferation of the ARPE-19 cells and BV2 microglial cells by these concentrations of bevacizumab (see online supplementary figure S1). In the present study, we also measured the levels of CNTF, which is a member of the interleukin-6 (IL-6) cytokine family. CNTF has been implicated in the development, differentiation and survival of retinal neurons. No changes in CNTF were observed in our IF study (figure 2C, E) or in our in vitro ARPE-19 cell study (figures 3 and 4) after bevacizumab treatment. However, BV2 microglia cells significantly expressed CNTF after bevacizumab treatment (figures 3 and 5). We speculate that this difference likely occurred because CNTF is a factor that is specifically secreted from microglia to affect retinal pigment epithelial cell proliferation. ${ }^{28} 29$

The process of membrane formation and proliferation is characterised by the migration and proliferation of various cell types, including retinal pigment epithelial cells, glial cells and myofibroblast-like cells of unknown origin. These cells organise into the proliferative membrane. ${ }^{30}{ }^{31}$ Retinal pigment epithelial cells are a principal source of growth factors that are involved in 
A

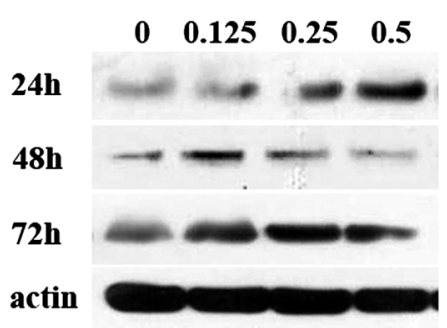

B

$\begin{array}{llll}0 & 0.125 & 0.25 & 0.5\end{array}$

24h

48h

72h

actin

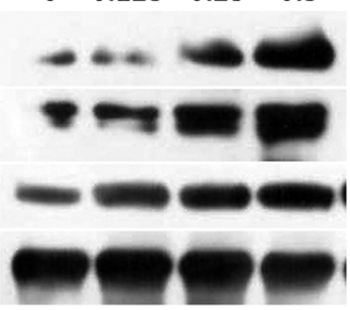

C

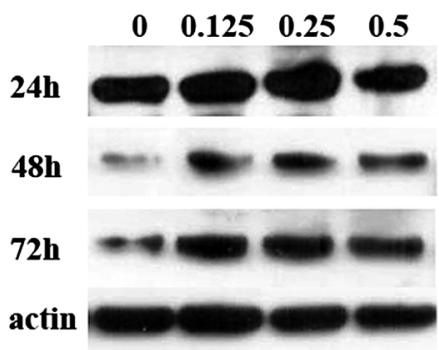

BV2 TGF beta

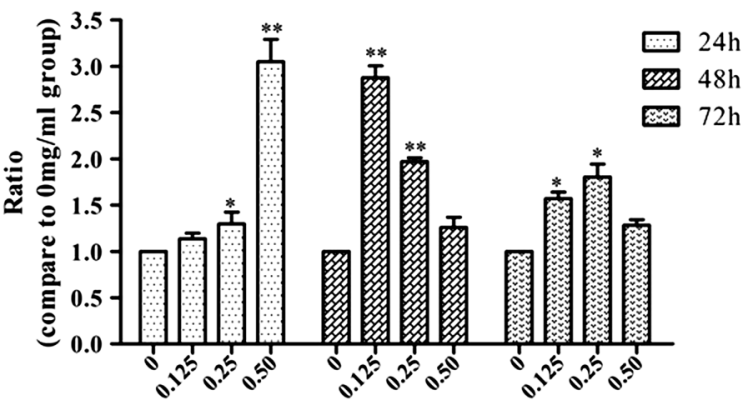

BV2 CTGF

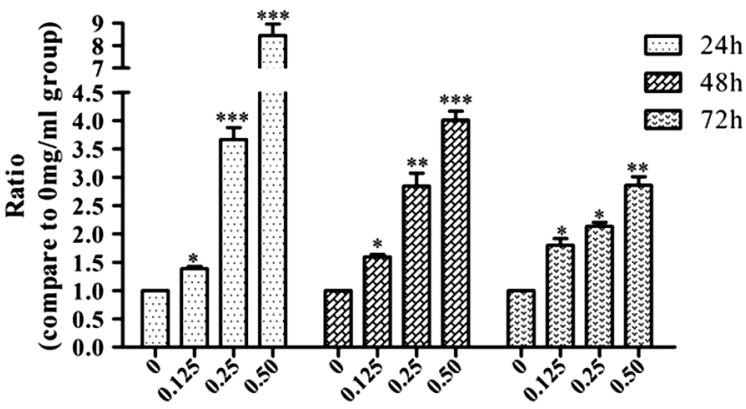

BV2 CNTF

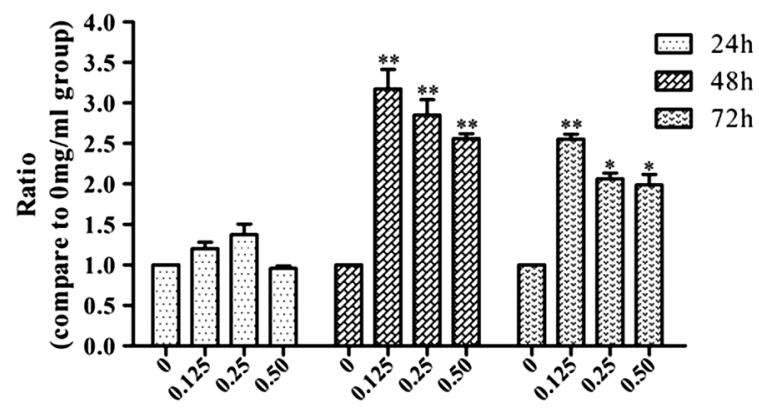

Figure 5 Western blot results of (A) transforming growth factor $\beta 2$ (TGFß2), (B) connective tissue growth factor (CTGF) and (C) ciliary neurotrophic factor (CNTF) expression in BV2 microglial cell lysates. Immunoblot images and statistical analyses for TGF 32 , CTGF and CNTF are presented. The results show that after intravitreal bevacizumab (IVB) injection, TGF $\beta 2$ and CTGF were upregulated at 24 and 48 hours but were reduced at 72 hours. CNTF expression was elevated at 48 hours and was sustained at 72 hours. Western blot analyses were repeated three times, and qualitatively similar results were obtained in each replicate. The data are presented as the means \pm SEM. ${ }^{*} p<0.05,{ }^{* *} p<0.01$.

the pathogenesis of fibrotic reactions inducing the epithelialmesenchymal transition, which leads to the progression of fibrotic tissue, retinal detachment and vision loss. Microglia are a common hallmark of various retinal diseases. ${ }^{32}$ Microglial function is kept in balance under normal conditions via crosstalk with other retinal cells, and microglia respond to different forms of retinal injury. ${ }^{32}$ The most recent data indicate that microglial activation is a common pathological mechanism in a variety of retinal diseases and that reactive microglia are neurotoxic and contribute to retinal degeneration and chronic parainflammation. Zeng et al ${ }^{11}$ thoroughly studied the characteristics of microglia in different stages of DR and identified sequential microglial responses during disease progression. Basic research results demonstrated that microglia are activated early after 4 weeks in STZ-induced DR rats and that high levels of TNF $\alpha$ and IL1 $\beta$ are observed in DR tissues. ${ }^{33}$ However, few studies have focused on the changes in microglia after anti-VEGF treatment. In the present study, we demonstrated that microglia secreted several growth factors, such as TGF 32 , CTGF and CNTF, after bevacizumab treatment, and this finding was consistent with previous results. ${ }^{34-36}$

There are several limitations to our study. First, considering the variation between different patients, the small number of cases was one of the limitations of the present study. Second, the microglial cell line that we used is of mouse origin rather than a human-derived or primary cell line, which weakens our results. However, similarly with previous research, the recombinant, humanised antibody bevacizumab still have interaction with BV2 microglial cells, even though weaker than human retinal pigment epithelial cells. ${ }^{37}$ Furthermore, we did not study the inflammatory cytokine levels after bevacizumab treatment, although these cytokines may be involved in the fibrosis process. However, our observations increase our understanding of the effects of bevacizumab treatment. 
Taken together, the preliminary results in the present study have several implications. (1) Anti-VEGF therapy may stimulate the secretion of several growth factors, such as TGF 32 , CTGF and CNTF. These cytokines may serve as novel therapeutic targets in the clinical management of PDR. (2) Understanding the timing of retinal fibrosis after anti-VEGF therapy before PDR is important. (3) Patients with PDR should be carefully assessed before treatment for characteristics including the existence of retinal fibrotic tissue, exudates and haemorrhages.

Acknowledgements The authors thank Jing Wen for her help with the immunostaining analysis.

Contributors $Y B$ and $X L$ : research design. $Q Z, Y Q, X S, Y B, W Y, L H$ and $Y J$ : experiments. $\mathrm{YB}, \mathrm{MZ}$ and $\mathrm{XL}$ : data analysis. $\mathrm{YB}$ and $\mathrm{QZ}$ : wrote or contributed to writing the manuscript. $\mathrm{YB}$ and $\mathrm{XL}$ : reviewed the manuscript. $\mathrm{YB}$ and $\mathrm{XL}$ : grant obtained.

Funding This work was supported by the Beijing Nova Program (Z131102000413004), and the National Natural Science Foundation of China (Grant 81570858).

Competing interests None declared.

Patient consent Obtained.

Ethics approval The Ethical Committee and Institutional Review Board of Peking University People's Hospital.

Provenance and peer review Not commissioned; externally peer reviewed.

\section{REFERENCES}

1 Penn JS, Madan A, Caldwell RB, et al. Vascular endothelial growth factor in eye disease. Prog Retin Eye Res 2008;27:331-71.

2 Olsson AK, Dimberg A, Kreuger J, et al. VEGF receptor signalling-in control of vascular function. Nat Rev Mol Cell Biol 2006;7:359-71.

3 Mintz-Hittner HA, Kennedy KA, Chuang AZ, et al. Efficacy of intravitreal bevacizumab for stage 3+ retinopathy of prematurity. $N$ Engl J Med 2011;364:603-15.

4 Martin DF, Maguire MG, Ying GS, et al., CATT Research Group. Ranibizumab and bevacizumab for neovascular age-related macular degeneration. N Engl J Med 2011:364:1897-908

5 Das A, McGuire PG, Rangasamy S. Diabetic macular edema: pathophysiology and novel therapeutic targets. Ophthalmology 2015;122:1375-94.

6 Cheung N, Mitchell P, Wong TY. Diabetic retinopathy. Lancet 2010;376:124-36.

7 Martinez-Zapata MJ, Martí-Carvajal AJ, Solà I, et al. Anti-vascular endothelial growth factor for proliferative diabetic retinopathy. Cochrane Database Syst Rev 2014;(11):CD008721.

8 Doguizi S, Ozdek S. Pigment epithelial tears associated with anti-VEGF therapy: incidence, long-term visual outcome, and relationship with pigment epithelial detachment in age-related macular degeneration. Retina 2014;34:1156-62.

9 van der Reis MI, La Heij EC, De Jong-Hesse Y, et al. A systematic review of the adverse events of intravitreal anti-vascular endothelial growth factor injections. Retina 2011:31:1449-69.

10 Strauss 0 . The retinal pigment epithelium in visual function. Physiol Rev 2005:85:845-81.

11 Zeng HY, Green WR, Tso MO. Microglial activation in human diabetic retinopathy. Arch Ophthalmol 2008;126:227-32.

12 Zhang $\mathrm{M}$, Chu $\mathrm{S}$, Zeng $\mathrm{F}$, et al. Bevacizumab modulates the process of fibrosis in vitro. Clin Experiment Ophthalmol 2015:43:173-9.

13 Van Geest RJ, Lesnik-Oberstein SY, Tan HS, et al. A shift in the balance of vascular endothelial growth factor and connective tissue growth factor by bevacizumab causes the angiofibrotic switch in proliferative diabetic retinopathy. $\mathrm{Br} J$ Ophthalmol 2012:96:587-90
14 Presta LG, Chen H, O'Connor SJ, et al. Humanization of an anti-vascular endothelial growth factor monoclonal antibody for the therapy of solid tumors and other disorders. Cancer Res 1997;57:4593-9.

15 Batman C, Ozdamar Y. The relation between bevacizumab injection and the formation of subretinal fibrosis in diabetic patients with panretinal photocoagulation. Ophthalmic Surg Lasers Imaging 2010;41:190-5.

16 El-Sabagh HA, Abdelghaffar W, Labib AM, et al. Preoperative intravitreal bevacizumab use as an adjuvant to diabetic vitrectomy: histopathologic findings and clinical implications. Ophthalmology 2011;118:636-41.

17 Arevalo JF, Maia M, Flynn HW Jr, et al. Tractional retinal detachment following intravitreal bevacizumab (Avastin) in patients with severe proliferative diabetic retinopathy. Br J Ophthalmol 2008;92:213-16.

18 Jonas JB, Schmidbauer M, Rensch F. Progression of tractional retinal detachment following intravitreal bevacizumab. Acta Ophthalmol 2009;87:571-2.

19 Kuiper EJ, Van Nieuwenhoven FA, de Smet MD, et al. The angio-fibrotic switch of VEGF and CTGF in proliferative diabetic retinopathy. PLOS ONE 2008:3:e2675.

20 Saika S, Yamanaka O, Sumioka T, et al. Fibrotic disorders in the eye: targets of gene therapy. Prog Retin Eye Res 2008;27:177-96.

21 Paine SK, Basu A, Mondal LK, et al. Association of vascular endothelial growth factor, transforming growth factor beta, and interferon gamma gene polymorphisms with proliferative diabetic retinopathy in patients with type 2 diabetes. Mol Vis 2012:18:2749-57.

22 Hirsch L, Nazari H, Sreekumar PG, et al. TGF- $\beta 2$ secretion from RPE decreases with polarization and becomes apically oriented. Cytokine 2015;71:394-6.

23 Chen $X$, Xiao W, Liu X, et al. Blockade of Jagged/Notch pathway abrogates transforming growth factor $\beta 2$-induced epithelial-mesenchymal transition in human retinal pigment epithelium cells. Curr Mol Med 2014;14:523-34.

24 Klaassen I, van Geest RJ, Kuiper EJ, et al. The role of CTGF in diabetic retinopathy. Exp Eye Res 2015;133:37-48.

25 Abu El-Asrar AM, Van den Steen PE, Al-Amro SA, et al. Expression of angiogenic and fibrogenic factors in proliferative vitreoretinal disorders. Int Ophthalmol 2007:27:11-22

26 Abu El-Asrar AM, Imtiaz Nawaz M, Kangave D, et al. Osteopontin and other regulators of angiogenesis and fibrogenesis in the vitreous from patients with proliferative vitreoretinal disorders. Mediators Inflamm 2012:2012:493034.

27 Watanabe D, Takagi H, Suzuma K, et al. Expression of connective tissue growth factor and its potential role in choroidal neovascularization. Retina 2005;25:911-18

28 Xue W, Cojocaru RI, Dudley VJ, et al. Ciliary neurotrophic factor induces genes associated with inflammation and gliosis in the retina: a gene profiling study of flow-sorted, Müller cells. PLOS ONE 2011;6:e20326.

29 Li R, Wen R, Banzon T, et al. CNTF mediates neurotrophic factor secretion and fluid absorption in human retinal pigment epithelium. PLOS ONE 2011;6:e23148.

30 Kampik A, Kenyon KR, Michels RG, et al. Epiretinal and vitreous membranes: comparative study of 56 cases. 1981. Retina 2005;25(Suppl 5):1445-54.

31 Jerdan JA, Pepose JS, Michels RG, et al. Proliferative vitreoretinopathy membranes. An immunohistochemical study. Ophthalmology 1989;96:801-10.

32 Karlstetter M, Scholz R, Rutar M, et al. Retinal microglia: just bystander or target for therapy? Prog Retin Eye Res 2015;45:30-57.

33 Krady JK, Basu A, Allen CM, et al. Minocycline reduces proinflammatory cytokine expression, microglial activation, and caspase-3 activation in a rodent model of diabetic retinopathy. Diabetes 2005;54:1559-65.

34 Yuan L, Neufeld AH. Activated microglia in the human glaucomatous optic nerve head. J Neurosci Res 2001;64:523-32.

35 Miao J, Ding M, Zhang $A$, et al. Pleiotrophin promotes microglia proliferation and secretion of neurotrophic factors by activating extracellular signal-regulated kinase 1/2 pathway. Neurosci Res 2012:74:269-76.

36 Kuiper EJ, Witmer AN, Klaassen I, et al. Differential expression of connective tissue growth factor in microglia and pericytes in the human diabetic retina. $\mathrm{Br} J$ Ophthalmol 2004;88:1082-7.

37 Bock F, Onderka J, Dietrich $T$, et al. Bevacizumab as a potent inhibitor of inflammatory corneal angiogenesis and lymphangiogenesis. Invest Ophthalmol Vis Sci 2007;48:2545-52. 